\title{
Nature as quantum computer
}

\author{
David Ritz Finkelstein
}

\begin{abstract}
Set theory reduces all processes to assembly and disassembly. A similar architecture is proposed for nature as quantum computer. It resolves the classical space-time underlying Feynman diagrams into a quantum network of creation and annihilation processes, reducing kinematics to quantum statistics, and regularizing the Lie algebra of the Einstein diffeomorphism group. The usually separate and singular Lie algebras of kinematics, statistics, and conserved currents merge into one regular statistics Lie algebra.

Keywords: Chronon, quantum gravity, quantum logic, quantum set theory, quantum space-time.
\end{abstract}

\section{Quantum theories}

I once asked Jack Schwartz what the difference was between mathematics and physics. At the time both were just equation-juggling to me. He was strap-hanging homeward from Stuyvesant High School, where we had just met, and he answered by drawing a hat in the subway air with his free hand:

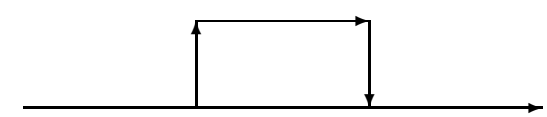

He explained that the bottom line is the real world and the top line is a mathematical theory. At its left-hand edge we take data from the real world and put them into a mathematical computation, and at the right-hand side we compare the output of the computation with nature. The loop closes if the theory is right.

This diagram also applies to quantum systems, if the statistical nature of quantum theory is taken into account. Then the bottom line is not one experiment on the system but a statistical population of them.

The question remains of what the symbols of mathematics mean to a mathematician. Some decades later I asked Jack Schwartz what "1" means, and he replied that it means itself. This took me aback. I had not considered that possibility. Symbols generally mean something not themselves. Memorandum:

$$
1=“ 1 ” .
$$

David Ritz Finkelstein

Georgia Institute of Technology, Atlanta GA 30342, e-mail: finkelstein@ gatech.edu 
After mathematizing aspects of economics, robotics, computing, relativity, and even knots, with a theory that predicted when ropes would slip on capstans, Jack Schwartz turned to the problem of putting quantum theory on firm foundations. His concern was not with mathematical rigor but physical. In a paper on "the pernicious influence of mathematics on science", obviously conversing with Wigner's famous lecture at the Courant Institute on "the unreasonable effectiveness of mathematics in the natural sciences" [16], Schwartz wrote:

The mathematical structure of operators in Hilbert space and unitary transformations is clear enough, as are certain features of the interpretation of this mathematics to give physical assertions, particularly assertions about general scattering experiments. But the larger question here, a systematic elaboration of the world-picture which quantum theory provides, is still unanswered. Philosophical questions of the deepest significance may well be involved. Here also, the mathematical formalism may be hiding as much as it reveals. [11]

I respond to this call here, though my answer might not be acceptable to him. He takes it for granted that quantum theory provides a mathematical world-picture, faithful or not, as classical physics did. It is not clear how literally he intended this. Some writings on physics assume that there are complete world pictures; in Gödel's sense of deciding all well-formed questions, not Bohr's weaker one, of answering all physical questions that can be answered. Bohr's "complete" is von Neumann's "maximal". One of the critical differences between quantum and classical physics is that quantum physics denies the existence of complete world pictures, yet asserts the existence of merely maximal ones. Perhaps mathematics is the most "pernicious influence" when it has been the most "unreasonably effective."

Classically, a mathematical model of a physical system is an isomorphism between physical predicates about the system and mathematical predicates about the model. Physical predicates are defined by physical processes of filtration or categorization. The stock example is a polarizing filter.

Boole already defined predicates by "acts of election". Mental predicates were mental acts in his theory; physical predicates are physical acts in quantum theory. There are then both input and outtake predicate logics, corresponding to input and outtake filtrations. These are dual lattices. They may be operationally defined as the Galois lattices of the relation "Inputs through filter $A$ do not trigger counters following filter B'.

Mathematical predicates, however, are defined axiomatically and form Boolean predicate lattices by fiat. Since models have Boolean predicate lattices and quantum systems have projective predicate lattices, the two cannot be isomorphic. Quantum systems do not have exact mathematical models because they have different logics than models.

Here the pernicious influence of mathematics is the highly infectious conviction that there must be an objective public reality, despite empirical evidence to the contrary. The mathematics of the quantum theory was invented shortly before the quantum theory was born, demonstrating the unreasonable effectiveness of mathematics. The quantum evolution has made mathematics more important for physics, not less. Mathematics still provides classical models for classical theories and now it also provides statistical models for quantum systems, in the following sense.

In classical mechanics a "state" completely describes the system, answering all experimental questions about it. Plato said clearly that what is real must have an objective state.

Quantum thought relinquishes this idea. This is no great loss, since we never came close to having the state of any physical system. When theorists speak of the state of Mars, they often mean its position and momentum, or possibly its orientation and angular velocity as well, ignoring trillions of coordinates of Martian atoms.

Some give the word "state" a new statistical meaning that can serve quantum theories, but the result has been continuing widespread confusion. We should keep the old meaning of complete information for "state" until the dust settles, so we can tell our students something important: quantum systems have none. 
A pure population is one that cannot be imitated by mixing statistically distinguishable ones. It goes with an atom of the lattice of predicates. Classically, a pure state, when such exist, is described by a probability distribution supported by one point: pure implies complete. A classical point particle with continuous coordinates, however, already has no pure state, because a point in a homogeneous continuum has probability 0 .

In quantum theory, all systems admit pure probability distributions, represented by points in a projective geometry, of probability measure 1 , not 0 ; and in analytic projective geometry, by a ray $\{\lambda \psi\}$ in a linear space $\mathscr{V}$ that we associate with the system.

Von Neumann was drawn to projective geometries that have a continuous range of dimensionalities. They can have no singlets. This ignores the problem of the infinities, of which singlets are the solution. Classical space-time already has no singlets.

As Malus found for linear polarization, the transition probability from one predicate ray to another is the squared cosine of the angle between them, almost never 0 or 1 . Heisenberg therefore called a vector with this probabilistic interpretation a "probability vector". Its components are probability amplitudes, their squares are relative probabilities. Quantum logic is a square root of classical logic.

But a lone classical system has no probability distribution, nor has the individual quantum system. Schrödinger and many others believed, at least at first, that a vector $\psi$, up to a factor, exists and evolves in a single quantum experiment, and that it was the state of a real physical object, a wave running around the atom. For brevity, call holders of this ontological interpretation "wavers", and holders of Heisenberg's more pragmatic one "chancers". Since the state of a real wave is indeed a wave-function. the term "wave-function" is a Trojan horse, smuggling waves into the camp of the chancers.

On the other hand, a population of similar experiments has at least two probability vectors or distributions, one for the input operation and a dual one for the outtake operation. To cope with this ancient duality wavers say that "the state vector collapses" from one to the other during a measurement. This locution is not part of Heisenberg's or Kolomogorov's probability theory, for a probability distribution or vector, be it classical or quantum, is unaffected by what happens to one member of its statistical population. It is not necessarily part of von Neumann's system either, since it was contributed to his book on quantum mechanics by a friend, and does not seem to occur in his later writings. When we interact with one quantum in a statistical population, we may wish to transfer the quantum to another statistical population but neither probability distribution changes; the quantum does.

Quantum theory eliminated what had been taken for granted: the possibility of a mathematical model of the physical system. Some classical conceptions assumes a complete picture of nature, as though taken from a preferred viewpoint outside nature. The corresponding quantum conception would be a plurality of partial representations of interactions with small parts of nature.

It is not obviously possible to visualize an atom completely, since on the atomic scale photons are not immaterial messengers but massive projectiles. Receiving them changes us, so emitting them must change their sources as much. On the atomic scale this reaction by the atom is greater than the action upon us is in the human scale. The smaller the system, the more its perception exhibits hysteresis, memory, non-commutativity. Moreover, we see one atomic transition by absorbing one photon that we cannot share. Quantum perception is ultimately private as well as non-commutative.

Yet all these impediments to depiction could have classical models. They make room for quantum theory, but they do not determine its specific features. If it is obvious that complete descriptions are impossible, it is amazing that maximal descriptions are possible. Partially order classes (predicates) by proper inclusion, and call classes that are $n$ steps away from the empty class, $n$-plets. The points of the projective geometry are the singlets the maximal descriptions of the quantum theory.

A classical doublet includes exactly two singlets. It cannot have a continuous symmetry group, only $S_{2}$. 
A quantum doublet has a theoretical infinity of statistically distinguishable singlets and an $\mathrm{SO}(2)$ symmetry.

Before studies of polarization, no physicist came close to a singlet of any system; Newton prepared polarization singlets with his crystals of Iceland spar. It is likely that when we reach the bottom of the world, we will find polarizations and spins on the beach; not strings, which have no singlets.

Heisenberg called his probabilistic brand of physics non-objective; it does not represent objects but laboratory actions and their probabilities. In the language of categories, a classical system can be presented as a category, whose objects are its states. There is a category of quantum systems too, but one quantum system is not a category, precisely because it has not enough objects (states, identities) in the categorical sense. Instead it is represented by an operator algebra, and only statistically.

"Philosophical questions of the deepest significance" are indeed involved. Jack Schwartz agreed with Kolmogorov that probability was not objective, that physics ought to be objective, and that therefore probability had no role in a fundamental physical theory. Some physicists who use quantum theory to great effect declare that they do not understand it, and expect it to devolve into a more objective theory. This likely results from a deep philosophical preference for objects, which are supposed to be knowable "as they are". This may be a pernicious influence of the unreasonable success of classical mathematical models in astronomy. But it might be innate, if we are hard-wired to see objects.

Exercises in physics often give a complete mathematical model of the system. This does not prepare the student for quantum physics. No one has ever encountered anything near a complete description of any physical system, classical or quantum. If quantum theory is right, there are none. Those who study physical systems only through such mathematical models may find it absurd and incomprehensible to say that physical systems have none.

Again, some note that quantum theory is "merely" instrumental, and find it unsatisfactory on that ground. This conveys more about the critic's philosophy than about the quantum theory. A Beethoven score too is "merely" instrumental. Physics is a performing art, and physicists are the performers, not the spectators; experimenters, not observers. The relation of a physical theory to physics is that of a menu to a meal. It is natural but naive to think that anything in nature has a complete objective description; naive in the sense that throwing a bottle at a villain on a movie screen is naive.

Again, Heisenberg has been criticized by wavers for providing no mathematical description of the measurement process in his theory; despite the fact that his main point is that none exists.

We can view any regular quantum system and its co-system as a quantum digital computer and its user. The quantum universe as computer system I sometimes call Qunivac for short. Qunivac differs crucially from artificial computers, however: It has no fixed hardware; it is all quantum jumping. It includes both computer and user. The interface between them is relatively fixed in artificial computers, but highly movable in Qunivac. Science is a Promethean attempt to hack into Qunivac.

\subsection{Canonical quantum theories}

Classical momentum and position coordinates commute: $p q-q p=0$. Classical physicists were never aware of this as a physical hypothesis; it seems to have been an unconscious assumption. We first became conscious of this assumption when Heisenberg corrected it to $\breve{p} \breve{q}-\breve{q} \breve{p}=i \hbar$. This has led physicists to make other unconscious assumptions conscious, and test them.

One operational meaning of this non-commutativity is that filters defining predicates about $p$ and $q$ do not commute. Such quantum or non-commutative logic was found in the laboratory by Newton for polarizations 
of light corpuscles, and was described in a quantum-theoretical way by Malus, who unwittingly used a twodimensional real Hilbert space of linear polarizations. When Boole first axiomatized what eventually became Boolean algebra, he noted excitedly that such a non-commutative logic was possible, without bringing up Newton's polarizers.

\subsection{Regular quantum theories}

In kinematics we represent our physical operations on the system by operators on a space of probability vectors. A regular quantum system is one with a finite-dimensional probability vector space, like a spin or a system of spins [2]. Its Lie algebra is simple. Its observable or normal operators have finite spectra. A regular theory still mentions infinities, such as the real number system $\mathbb{R}$, but these result from regarding the co-system as infinite and are harmless if we abstain from questions about the co-system.

\section{Yang space-times}

Quantizing space-time to avoid infinities was proposed in about 1930 by Heisenberg, Ivanenko, and others. The first example was provided by Snyder in 1947 and its commutation relations were immediately simplified by Yang to those of $\mathfrak{y}:=\operatorname{so}(5 ; 1)$, precisely for the sake of simplicity [17]. A Yang space-time (in the general sense) is one whose orbital variables span a semisimple Lie algebra, called the Yang Lie algebra.

Yang so $(5,1)$ is not conformal so $(5,1)$. It defines a quantum space, while conformal so(5; 1) acts on a classical one.

Snyder and Yang did not complete their regularizations but continued to represent their Lie algebras in the singular su $(\infty)$ of Hilbert space.

Earlier, R. P. Feynman had quantized space-time by replacing continuous space-time coordinates by sums of Dirac spin operators (apparently unpublished), which also leaves Hilbert space; though he broke off this work in an early phase to study the Lamb shift for Bethe. Feynman quantized space-time but not momenta. The Yang model quantizes space-time and momentum-energy, but is still singular. The Penrose position vector $\mathbf{x}$ is a finite sum of Pauli spin operators; the momentum vector is not represented.

Here are the quantum space-time variables of Feynman, Yang, and Penrose, in quantum units:

$$
\begin{aligned}
& \text { Feynman [4] } \delta \check{x}^{\mu}=\gamma^{\mu} \text {, } \\
& \text { Yang [17] } \quad \check{x}^{\mu}=L^{5 \mu}=i \eta^{[5} \partial^{\mu]}, \check{p}_{\mu}=L_{6 \mu}=i \eta_{[6} \partial_{\mu]} \text {. } \\
& \text { Penrose [9] } \delta \check{x}^{k}=\sigma^{k} \text {, }
\end{aligned}
$$

In such theories, particles do not define irreducible unitary representations of the Poincare group as Wigner proposed, but irreducible normal representations of a slightly different simple Lie algebra. The physical constants of the Feynman or Yang groups are the speed and action units $c, \hbar$ of earlier group decontractions, with additional elementary time and energy units $\mathrm{X}$ and $\mathrm{E}$ with $\mathrm{XE}=\hbar$, and a huge integer $N$. The quantum units $X$ of time and $E$ of energy are here called the chrone and the erge; it is not yet clear whether these are the Planck units. Under the Yang relativity group, time in chrones in one frame is just energy in erges in another; time converts into energy, mass. The conversion factor is a huge quantum of 
power, one erge per chrone, perhaps the Planck power. The conversion is not easy but requires melting the vacuum organization that distinguishes energy and time.

The synthesis described here is finite-dimensional, quantizes all the orbital and field variables, and includes spin. The other internal coordinates of the Standard Model are easily tacked on, but it would be disappointing if the quantization of space-time did not lead us to a deeper synthesis of the internal variables.

Einstein's local equivalence principle again suggests that space-time must be quantized. Following Galileo, Einstein equates a gravitational field and an acceleration. Since the field is quantized, so is the acceleration. This is a second time derivative of spacial coordinates with respect to time; if the space and time coordinates were commutative, the acceleration would be too.

Since $i$ and $-i$ are interchanged by Wigner time reversal, they can be regarded as two values of a discrete dynamical variable $i$ that happens to be central. This centrality makes the Heisenberg Lie algebra singular and leads to infinities. The Yang simplification suspended the centrality of $i$. It is therefore a real quantum theory of the Stückelberg kind [15]. For correspondence with the standard complex theory, Yang provides a quantized imaginary $\check{l} \in \mathbb{S}$ whose classical correspondent is $i$, with $\breve{l} \circ i$, but a self-organization must be invoked to single one $i$ out of many possibilities.

The Standard Model spinlike groups can all be defined by their actions on a probability vector space of about 16 dimensions. The orbital group seems to act on a much larger number of dimensions $N \gg 16$. Therefore events of history are not randomly scattered but highly organized locally into something like a thin truss dome of four longitudinal dimensions and several short transverse dimensions. This dome must support the particle spectrum, sharp bands of highly coherent transmission, and so is presumably crystalline, as Newton inferred from transverse photon polarization.

Regular space-times call for regular Lie algebras. Here is one suggested by those of (3), based on the contraction $\operatorname{spin}(3,3) \circ \mathfrak{h p}(3,3):$

$$
\begin{aligned}
\check{x}^{\mu} & =L^{5 \mu}=\bar{\psi} \gamma^{[5 \mu]} \psi, \\
\check{p}_{\mu} & =\quad L_{6 \mu}=\bar{\psi} \gamma_{[6 \mu]} \psi, \\
\check{i} & =\mathrm{N}^{-1} L_{65}=\bar{\psi} \gamma^{\top} \psi .
\end{aligned}
$$

Here $\psi$ is a chronon IO operator and $\bar{\psi}=\beta \psi$ is its Pauli adjoint. The combination $\bar{\psi} \ldots \psi$ is the usual covariant accumulator, summing many replicas of its argument with attention to polarity. The eight $\gamma^{v}$ generate the Clifford algebra of $\operatorname{spin}(4,4)$, with top (volume) element $\gamma^{\top}=\gamma^{8} \ldots \gamma^{1}$.

\section{Whither physics?}

First the ancient axioms of space and time failed us in physical experiments and then the axioms of Boolean logic. There are now well-known physical geometries and lower-order physical logic side by side with the older mathematical ones. Higher-order logic is evidently next in line to join the empire of the empirical.

Define an operational theory of a system as a semigroup whose elements are the feasible operations on the system by the co-system (the rest of the cosmos, including us), provided with their probabilities. The kinematics gives the possibilities, the dynamics attaches probabilities.

In quantum theories, each operation is represented by a projective transformation of a specified projective geometry whose points are singlet quantum input or outtake operations. A special conic section in the projective geometry defines transition probability amplitudes. 
The first-order logic of the system is the sub-semigroup of filtration operations. The set theory of the system deals with operations of system assembly and disassembly.

Our operations ordinarily rely on the organization of the co-system, natural or artificial, as do operating manuals or cookbooks. Highly organized complex elements of the co-system may enter the system theory only through several of their many parameters.

Thus the idea of a Universal Theory is absurd. Measurements do not bring us ever closer to Truth, but invalidate earlier facts as fast as they validate new ones, and omit much about the co-system. A dynamical law cannot be universal if it is overridden whenever we measure anything.

Then what shapes our course? Two processes by which physical theories evolve correspond to biological evolutionary processes studied by Charles Darwin and Lynn Margulis. The Darwinian one is implicit in Yang space-time and explicit in work of Segal: Physical theory evolves towards semisimple Lie algebras [12].

Almost all Lie groups have regular Killing forms. A singular Killing form is a very rare fish; the least change in its structure tensor can regularize it. Singularity is structurally unstable. As measurements of structure constants improve, a singular Lie algebra has survival probability 0 relative to its regular neighbors, which outnumber it $\infty$-to- 1 . This is the Darwinian argument for semisimplicity.

Gerstenhaber, influenced by Segal, described homologically a rich terrain of Lie groups connected by contractions that carry groups out of stable valleys of simplicity, along ridges between the valleys, and up to singular peaks [6]. According to the simplicity principle, physics today is a brook flowing down the simplicity-gradient to some green valley in Gerstenhaber-land.

Thus the Galileo Lie algebra lies on a singular ridge between the valleys of Lorentz so $(3,1)$ and Euclidean so(4). Poincaré iso $(3 ; 1)$ perches on a higher singular ridge between the valleys of deSitter so(3;2) and so $(4 ; 1)$.

The special relativity Lie algebra iso $(3,1)$ makes the observer a rigid body with 10 degrees of freedom, like a speck of diamond dust. The general relativity group Diff, however, makes the observer an infinite squid, unaffected by gravity, crossing all horizons freely, continuously deformable without limit. This surely overcompensates. It still works at the level of astronomy, and it greatly influenced the Weyl, Yang-Mills, Schwinger, and Standard Model theories of gauge. Yet general relativity is much more singular than special relativity, and so disproves the Segal theory of evolution as regularization.

The Margulis evolutionary process is expressed in biology by symbiogenesis [7] and in technology by modular architecture [13]. In modular evolution, two modules of some complexity unite with each other to form a more complex system that can use survival strategies of both.

To cope with complexity, physics has united singular modules rather than wait for regular ones. Stability and complexity are both vital for theories, and they pull in opposite directions at present. They must be harnessed to pull together. General covariance cannot be right in its present form. Its group Diff(4) too must be de-contracted.

The de-contraction of Diff should be compatible with the Yang de-contraction of the Heisenberg-Poincaré Lie algebra $\mathfrak{h p}(3,1) \leftarrow Y$. Here $Y$ is a high-dimensional orthogonal representation $R: \mathfrak{y} \rightarrow \operatorname{so}(N), Y=R(\mathfrak{y})$, of the simple Yang Lie algebra $\mathfrak{y}$. The most obvious candidate for the de-contracted diff is some $\operatorname{so}(N)$, assuming a commutative diagram of Lie algebra transformations 


$$
\begin{array}{cccc}
Y & \hookrightarrow & \mathfrak{h p}(3,1) \\
\downarrow & & \downarrow \\
\operatorname{so}(N) & \hookrightarrow & \operatorname{diff}(4)
\end{array}
$$

Gauge theories like gravity theory combine identical gauge modules at every point of space-time. This is a quantification too. It brings together three Lie algebras: a kinematic one for orbital variables, a statistical one for the gauge vector quanta, and a gauge Lie algebra for conserved currents. All need regularization. In a quantum set theory, all operations are reduced to assembly and disassembly. Kinematics is all statistics. The three singular Lie algebras must then become aspects of one regular one.

\section{Below Hilbert space}

Canonical quantization uses an infinite-dimensional Hilbert space $\mathscr{H}$ of probability vectors. This space is still too weak and already too large for quantum theory.

Too large, in that there are infinitely more orthogonal rays in $\mathscr{H}$ than there can be disjoint pure populations in any physical laboratory. This makes its unitary Lie algebra singular and leads to divergent sums.

Too weak, in that $\mathscr{H}$ lacks fundamental concepts of modular structure and interaction. True, it can represent general actions; but under close inspection all actions resolve into interactions. Hilbert space has to become smarter to express these.

It does not seem that a quantum set theory closely modeled on classical set theory will work for quantum physics as classical set theory works for classical physics [5]. The main problem is that set theory formulates "laws of thought", not of physics. A set is a collection "thought of as one". A proton's position and spin are usually united by bracing, as one unites sets, but presumably they are not held together by our thinking of them "as one". Higher-order logic does not have operational meaning as the first-order logic does.

Regard Cantor and Peano as proposing basic vertices $x \in y$ and $y=\{x\}$, respectively, for the graph (category) of all mathematical objects. Quantum theory must replace these ideational vertices by operational ones adequate for the non-category of physical processes $(\S 9)$. The result does not resemble set theory enough to warrant the name.

\section{Quantification}

Quantification is a logical process that turns a theory of an individual into a theory of a multiplicity of like individuals. Set theory iterates it. The logician William Hamilton introduced the term in 1850. It has two famous quantum correspondents: Fermi quantification is regular, and Bose quantification is singular but is regularized by Palev $(\S 6)$.

Quantization and quantification traverse the same road in opposite directions. Quantification assembles individuals into an individual of higher rank. Quantization resolves an individual into individuals of lower rank. Quantization introduces a quantum constant when it begins from a classical limit in which that constant 
has approached 0. In quantification a quantum constant provided by the lower-rank individual vanishes in a singular limit. A "second quantization" is well-known to be a quantification, but it is also a "second quantification" since it follows a quantization, which implies a first quantification. In this project we express all quantizations, including Yang space-time quantization ( $(2)$, as inverse quantifications, to arrive at the modular architecture of the quantum universe. All kinematics is statistics.

The Standard Model uses bracing or uniting operation $\{a, b, \ldots\}$, at least tacitly, to assemble quanta from their various conceptual parts: orbital, spin, isospin, color, and so forth. The lowest-order predicate algebrahas been quantized. The higher-order set theory rests on the lower-order; it must be quantized or dropped out of physics. Here we quantize a small part of it and drop the rest.

Cantor intended to represent the workings of the infinite Mind of God, while physicists seek merely to represent the workings of finite quantum systems. For finite algebraic purposes, Peano's one-to-one uniting operation $y=\imath x=\{x\}$, sometimes written $\bar{x}$ here, will do as the key construct of a truncated set theory. $l$ turns what it touches into a monad, a unit set. Polyads are built from monads by disjoint union $a \vee b$. For example $\{a, b\}:=\imath a \vee \imath b$ is a dyad; not to be confused with a doublet. An $n$-ad is a product of $n$ factors; an $m$-tuplet is a sum of $m$ terms.

Uniting (bracing) occurs in many important constructs of the Standard Model and gravity theory. It is used to suspend associativity of the tensor product. For example it associates spin variables with their proper orbital variables. Again, a hadron is a triad of quarks, and so the triads must be united to associate their quarks properly when a pair of hadrons forms a deuteron. This hierarchy of unitings is commonly tacit. It will be hard to do without it. But bracing violates operationality as much as (say) absolute rest.

Let

$$
\mathbb{S}=2^{\mathbb{S}}=\exp _{2} \mathbb{S}
$$

designate the group of perfinite sets (sets that are ancestrally finite, hereditarily finite, finite all the way down). $\mathbb{S}$ is an infinite $\mathbb{N}$-graded group generated recursively from the empty set 1 by

- the monadic uniting operation $l x$; and

- the dyadic product operation $x \sqcup y=x$ XOR $y$, for the symmetric union or XOR.

All $x \in \mathbb{S}$ obey the Clifford-like rule

$$
x \sqcup x=1 .
$$

$\mathbb{S}$ is $\mathbb{N}$-graded by cardinality ("adicity"). $\mathbb{S}$ is also $\mathbb{N}$-graded by rank, the number of nested $\imath$ operations. $\mathbb{S}$ also has a product $x \vee y=x$ POR $y$, the Peircian or partial OR, determined by $\sqcup$. POR formalizes Boole's original partial-addition operation $\dot{+}$ and obeys the Grassmann-like rule

$$
x \vee x=0 .
$$

This 0 is the OM (for $\Omega$ ) of Jack Schwartz's programming language SETL: a space-filler indicating the intentional omission of any meaningful symbol. It is the semantic 0 .

A plausible probability space for quantum sets is a linearized $\mathbb{S}$,

$$
\check{\mathbb{S}}=\check{2} \check{\mathscr{S}}=\exp _{\check{2}} \check{1} \check{S}
$$

the least linear space that is its own Clifford algebra. It is generated recursively from the linear space $\mathbb{R} \subset \breve{S}$ representing the empty set by three operations:

- a monadic uniting operation $\imath: \breve{\mathbb{S}} \rightarrow \breve{\mathbb{S}}$;

- a dyadic Clifford product $x \sqcup y$, sometimes written $x y$, for the symmetric union; and 
- the dyadic addition operation $x+y$ for quantum superposition

$\breve{S}$ also has a Grassmann product $x \vee y$ determined by $\sqcup$. For all $x$ in a certain basis called classical, $x \sqcup x= \pm 1$ and $x \vee x=0$, as in the classical theory and with the classical meanings.

$\breve{\mathbb{S}}$ is $\mathbb{N}$-graded by a cardinality operator Grade $\breve{S}$ is also graded by the operator Rank, the number of nested $\imath$ operations.

A physical theory needs only a finite-dimensional probability tensor space, but it is convenient to keep $\breve{S}$ infinite-dimensional so that it also contains the singular limits presently in common use.

\section{Palev statistics}

Palev regularized quantum statistics [8] as Yang regularized quantum kinematics [17]: by de-contracting a singular Lie algebra into a nearby regular one.

In an even statistics of Palev type $\mathfrak{p}$ [8],

1. $\mathfrak{p}$ is a classical (simple) Lie algebra.

2. The probability vector space of the individual quantum is $\mathfrak{p}$.

3. The probability algebra of the aggregate is $\mathscr{P}=$ poly $\mathfrak{p}$, an algebra of non-commutative polynomials over $\mathfrak{p}$ identified modulo the commutation relations of $\mathfrak{p}$, defining a representation of $\mathfrak{p}$.

Palev also considers mixed even and odd statistics, where $\mathfrak{p}$ is a Lie superalgebra. In the present instance $\mathfrak{p}=$ $\operatorname{so}(N, N)$ with $N \gg 1$. Bose statistics is merely a useful singular limit of an even Palev statistics, ultimately unphysical [5].

There is no physical boundary between statistics and kinematics ( $(5)$, only a historical one. It is natural to regularize both at once. Di-fermions obey a Bose statistics only approximately, a Palev statistics exactly. The Palev Lie algebra can even be the Yang Lie algebra.

A brief review: The three-dimensional Heisenberg Lie algebra $\mathfrak{h}(1)$, with the singular canonical commutation relation

$$
\mathfrak{h}(1): \quad[q, p]=i \hbar,
$$

underlies both quantum oscillator kinematics and Bose statistics. $\mathfrak{h}(1)$ lies on the ridge between $\operatorname{spin}(2,1)$ and spin(3). Quantum relativity needs an indefinite metric, so choose the indefinite case in this toy example:

$$
\operatorname{spin}(2,1): \quad[q, p]=r, \quad[p, r]=q, \quad[q, r]=p
$$

To contract $\operatorname{spin}(2,1)$ to $\mathfrak{h}(1)$, the variable $r$ must freeze to a central imaginary as the dimension $D$ of the representation goes to infinity: $r \approx N i$, where $N \rightarrow \infty$ with $D$. Call such a process an "organized singular limit" and write, for example,

$$
\operatorname{spin}(2,1) \circ \mathfrak{h}(1), \quad \check{q} \leftrightarrow q, \quad \check{p} \circ p, \quad \check{r} \circ \mathrm{Ni} .
$$

The circle in " $\circ \rightarrow$ " represents the regular algebra, the tip of the arrow the singular one, and the connecting line the self-organization, if any, and the homotopy that connect the regular to the singular. 


\section{Neutral metrics}

The metric in the Clifford algebra must be specified. To represent in the one space $\breve{S}$ the duality between source and sink that each experimenter sees, the probability form should be neutral, like that of a quantum space in the sense of [10], and like the Pauli-Cartan metric $\beta$ of spinor space. To fix the sign convention: source vectors have positive norm, sink vectors negative.

Every finite-dimensional Grassmann algebra, and therefore every subspace $\breve{S}[r] \subset \breve{S}$ of finite rank $r$, has a natural neutral norm, the Berezin $L^{(2)}$ norm

$$
\|w\|:=\int d \gamma^{\top} w \vee w=\frac{d}{d \gamma^{\top}} w \vee w=\beta w w
$$

where $\gamma^{\top} \in \check{\mathbf{S}}[r]$ is a top Grassmann element, and $\beta w w$ is the polarization of $\|w\|$. The Berezin norm is identical to the Cartan norm for spinors.

There is a frame-dependent scalar factor in this top element, and therefore in the norm (13); but all physical quantities, which are of degree 0 in the norm, so this factor drops out.

The previous rank $\check{\mathbf{S}}[r-1]$ is an isotropic space of this norm, representing linear combinations of sinks and sources with equal probability.

The norm $\beta$ defines the Clifford product $x \sqcup y$ on $\breve{S}[r]$ by the Clifford rule

$$
x \sqcup x=\|x\|
$$

for vectors (of grade 1) and dual vectors (of grade Dim $\breve{S}[r]-1$ ). Here this is the exclusion principle.

In this quantum set theory as in classical set theory, any set can be in any set only 0 or 1 times. Multiple occupancy is forbidden by fiat. Sets with even statistics have to be pairs of monads. This set theory does not acknowledge elementary bosons, which violate Leibniz's principle that indistinguishable objects are one. Indistinguishable laser photons can be $10^{1} 0$ and more. The grade parity operator $g(x) \doteq 0,1$ of a set $x$ is the parity of the grade (= cardinality) of $x$. It defines the "statistics" of $x$; called even (or bosonic) if $g=0$, and odd (fermionic) if $g=1$.

The quantum set theory of $\breve{S}$ immediately conflicts with the Standard Model on the conservation of exchange parity (statistics). For any $x, y \in \breve{S}, \imath x$ and $t y$ are monadic (of grade 1) and so is their uniting $\{\{x, y\}\}=\imath(\imath x \vee \imath y)$. But in nature so far, as in the Standard Model, a composite of two odd quanta is always even. In nature, composition conserves grade parity but not in $\breve{S}$. The question was not considered explicitly in $\mathbb{S}$, which opted to deal in sets alone, although other modes of aggregation exist in classical thought. Sets are all of odd parity in that they obey the exclusion principle $x \vee x=0$. The functor Grass works well on the probability space of fermions. But its iteration Grass $^{2}$ violates the conservation of statistics (exchange parity $X=0,1)$.

Another problem with $\breve{S}$ as a paradigm is that its $\imath$ is infinitely reducible. This has classical roots. The classical $l$ is a formal sum of its restrictions $\imath^{(m)}$ to sets of cardinality $m$. Correspondingly, the quantum $l$ reduces to a sum

$$
\imath=\sum_{m} \imath^{(m)}
$$

of its projections on $m$-adics. In the classical basis $\left\{1_{s}\right\} \subset \breve{S}$, the operator $\iota^{(m)}$ has the matrix elements

$$
\imath^{\left\{s_{1} \ldots s_{m}\right\}}{ }_{s_{1} \ldots s_{m}}
$$


in which for any $s_{1}, \ldots, s_{m},\left\{s_{1} \ldots s_{m}\right\}$ is a single collective index of one higher rank than any of $s_{1}, \ldots, s_{m}$. The repeated unsummed indices break the linear group, but the tensor

$$
{ }_{i}{ }^{\left\{s_{3} s_{4}\right\}}{ }_{s_{1} s_{2}}:=\delta^{s_{3} s_{4}}{ }_{s_{1} s_{2}}
$$

is invariant under the linear group and reduces to $t^{(2)}$ in the classical basis. Such $t^{(m)}$ are the irreducible vertices of this quantum set theory. If they occur in nature, their occurrences supply their operational definitions. If they do not occur in nature, we have to remove them from the physical theory, by burying them in its infrastructure if necessary.

There is also a problem with relativity. Set theory takes the Eternal view and has a distinguished frame, while a quantum theory admits only the secular perspectives of many limited experimenters. $\mathbb{S}$ and $\breve{S}$ both have preferred frames.

Here $\breve{S}$ will be truncated to two ranks, the points and links required to build a network of interactions. The rest of the usual hierarchy of ranks is replaced by a hierarchy of clusterings of clusters in the network. The points have Fermi statistics, the links Palev.

\section{Quantum events}

Einstein understood an event to be a smallest possible occurrence, and took the collision of two small hard balls as an approximation to an event. Collisions of much smaller things have been studied since then, and they have more internal variables than Einstein's idealized buckshot, such as spins and Standard Model charges. It is remarkable that the Standard Model still uses the same mathematical representation of spacetime events that Einstein did. Is this unreasonable effectiveness or pernicious influence?

To infer new classical space-time dimensions from the internal quantum numbers is archaic today. Any classical description is a low-resolution many-quantum description. Quanta are not born out of continua; continua are assembled quanta. To be sure, the first quanta were explained by quantization. Similarly, Swift assures us, the first roast pig was discovered when a barn burnt down, and for some time, a barn was burnt down for every roast pig. The continua of string theory and of modern followers of Kaluza are our barns. Eventually it will be recognized, as Dirac did, that one can have a quantum without quantizing some continuum.

The charges of the elementary particles have small discrete spectra, in stark contrast to the quasicontinuous spectra of position coordinates. This tells us that the charge degrees of freedom do not organize themselves into quasi-continua as spins do in the models of Feynman and Penrose. Conceivably this is the salient difference between the charges and spin.

According to present physical theory, we never perceive space-time itself, only quanta. Quantizing spacetime is best understood as extending the familiar quantization of orbital angular momentum to the other orbital variables $x^{\mu}, p_{\mu}$, like Feynman, Snyder, Penrose, and Yang. It resolves a fine-structure of quanta that canonical quantum theories smear out. Since our measurements always concern quanta, we have no need for both quanta and space-time. Regard space-time as another classical reification, a mental extension of the solid laboratory floor beam to the distant stars.

Particle collisions take the place of Einstein's buckshot collisions. In the relativistic canonical quantum theory the 15 orbital operators of en event,

$$
x^{\mu}, p_{\mu}, L_{\mu^{\prime} \mu}, i \in \mathfrak{h p}(3,1) .
$$


span the singular Heisenberg-Poincare Lie algebra. In addition quanta have the internal variables of the Standard Model. To describe one quantum in a canonical theory it suffices to tensor-multiply certain of these spaces and unite the product with braces or $t$. In the canonical theory, however, collisions break up into several IO processes for quanta. One space-time quantization resolves these in turn into chronons two ranks of aggregation lower, the fundamental actions of the theory.

Since fermions exist, we cannot begin the inductive construction of the quantum universe as computer with the empty set alone, which is even. Begin with a foundation of $\mathrm{N}$ primal odd chronons represented by basis spinors $\chi_{a} \in \mathscr{X}, a \in \mathrm{N} \gg 1$. A set-theoretic fermion field hierarchically unites an odd number of chronons first into events $\varepsilon=\left\{\chi_{e} \vee \ldots \vee \chi_{e}\right\}$, and then into fields $\phi=\varepsilon_{a} \vee \ldots \vee \varepsilon_{b}$. To form a gauge boson, unite chronons into di-chronons, into gaugeon events, into a gaugeon field.

Set theory envisages the construction of its universe from the empty set in an infinity of ranks, by a Mathematician outside the theory. A similar construction within superset theory $\breve{S}$ requires on the order of 10 ranks or less to accommodate physics. It does not represent the entire universe, since mundane experimenters, like the extramundane Mathematician, are there from start to finish, controlling the system but largely undescribed in the theory.

Nevertheless it is advantageous for $\breve{S}$ to be infinite-dimensional. so that the singular limit of classical space-time can be carried out within $\breve{S}$.

Absolute Space and Absolute Time have left the theater but Absolute Space-Time remains in the Standard Model and general relativity. How did this myth begin?

Etymologically, a "line" is a linen string, a "point" is a puncture or stick, and "geometry" is earthmeasurement. Sticks connected by linen strings used to puncture the Nile flood-plain each spring. This is supposed to be the origin of geometry. These sticks, however, have position, momentum, angular momentum, time, and energy. Presumably these were first neglected and then lost on the way to Greece, giving rise ultimately to the constructs of space-time. In quantum field theory space-time is merely an index on some variables, part of the infrastructure. Space-time coordinates are actually carried by physical quanta, not by mythical space-time points.

The fundamental events in nature are then IO operations for quanta. In the Standard Model every fermion carries many variables: one hypercharge $y$, one generation index $g$, three isospin $\tau^{k}$, four Dirac $\gamma^{\mu}$, eight color charges $\chi^{c}$, four space-time $x^{\mu}$, four energy-momentum $p_{\mu}$, and six angular momenta $L_{\mu^{\prime} \mu}$. Like atomic number and atomic weight, these variables tell us something about the structure and composition of the fermion. We are to fit them all into a semisimple operator algebra $\mathscr{E}$ of event variables.

To recover the canonical quantum theory from a Yang spin $(3,3)$ theory, one freezes one rotational degree of freedom $L_{65} \circ \rightarrow N i$, as a step in the organized singular limit in which $\hbar, \mathrm{X} \rightarrow 0$ and $N \rightarrow \infty$. Like the Higgs and gravitational fields, $i$ is the non-zero vacuum value of a non-commutative field operator $\breve{l}=\mathrm{七}_{65} / N$ . Presumably all vacuum values result from freezing and self-organization. The possibility that the Yang $\check{l}$ is the Higgs field has not been excluded.

In the Standard Model, odd probability vectors form a Clifford algebra, even ones a Heisenberg Lie algebra, and orbital coordinates $x, p$ another Heisenberg algebra of lower rank. This singular structure is presumably a jury-rig. A nearby finite-dimensional algebra arises from the construction out of fermion pairs; not a Bose Lie algebra but a Palev one based on so $(N, N)$.

Since the noncompact Lorentz group will not fit into a compact unitary group, a finite-dimensional relativistic quantum theory has to renounce definiteness of the probability norm as well as the space-time norm. Relativistic spinor theory provides one resolution [3]. The space R of real Majorana Dirac spinors has no invariant definite metric form. Instead it has one Pauli form $\beta$ that is invariant but not definite, and a plethora of Hilbert forms $\mathrm{h}$ that are definite but not invariant, associated with different time axes; and nevertheless it works. 
Take spinor spaces as elementary building blocks, so that their aggregates inherit this multi-metric structure. Correspondence with present physics requires that a unique invariant global hermitian form $\mathrm{H} \leftarrow \mathrm{h}$ exist in the singular limit of classical space-time.

\section{Quantum gauges}

Experience severely breaks the kinematic symmetry between position and momentum in quantum mechanics. So does the locality principle, which requires field variables coupled in the Lagrangian or action to share a space-time point, not a point in the Fourier transform momentum-energy space. So does gauge field theory, including general relativity. We must resolve this discord between the diffeomorphism and canonical groups into a harmony; they work too well to be merely discarded.

Gauge theories today rest on the following relation, with readings depending on context; "energy" stands for "energy-momentum" here:

$$
\begin{array}{clcc}
D_{\mu} & =\partial_{\mu} & - & \Delta_{\mu}, \\
\text { covariant derivative } & =\text { Lie derivative } & - & \text { connection }, \\
\text { kinetic energy } & = & \text { total energy } & - \text { potential energy }
\end{array}
$$

In a Yang-Feynman quantum event space, the invariant concept is the Yang-Feynman quantized coordinate $\check{p}_{y^{\prime} y}$, This is a cumulation

$$
\check{p}_{y^{\prime} y}=\bar{\psi} \gamma_{y^{\prime} y} \psi
$$

of many spin-matrix terms $\gamma_{y^{\prime} y}$, generating a regular Lie algebra $\mathfrak{y}$. In turn, $\check{p}_{y^{\prime} y}$ has a singular semi-classical contraction $p_{y^{\prime} y} \in \mathfrak{h} \mathfrak{p}(3,1) \leftarrow \mathfrak{y}$, the usual 15 canonical quantum event coordinates $p_{y^{\prime} y}=\left(x^{\mu}, p_{\mu}, L_{\mu^{\prime} \mu}, i\right)$. Then underlying the usual gauge field kinematics (19) is the single-event relation

$$
\begin{aligned}
& \check{p}_{y^{\prime} y}=\begin{array}{lll}
p_{y^{\prime} y} & - & P_{y^{\prime} y},
\end{array} \\
& \text { Yang coordinate }=\text { canonical coordinate }- \text { quantum correction. }
\end{aligned}
$$

Both terms on the right-hand side are singular; only the left-hand side is physically meaningful. Accumulate this relation over all events in a field by a higher-rank cumulation $\check{\phi} \ldots \phi$ and we arrive at the regular correspondent of (19).

In older terms: classical gravitational curvature and the electromagnetic field are higher order corrections that remain when we contract quantum event space to a flat, field-free, classical space-time. They are classical effective descriptions of quantum non-commutativity at the chronon level.

C. S. Peirce noted that while only line graphs can be built up from a 2-vertex alone, the most general graph can be simulated with a triadic vertex alone. The natural physical candidate for a universal vertex is indeed triadic, that of gauge physics, with three limbs: a chiral spinor $\psi$, its dual $\bar{\psi}$, and a gauge vector boson (actually, palevon) $\phi$ :

$$
\bar{\psi} \gamma^{m} A_{m} \psi=: \bar{\psi} \phi \psi=\gamma^{y^{\prime \prime} y^{\prime} y} \bar{\psi}_{y^{\prime}}, \phi_{y^{\prime}} \psi_{y} .
$$

Here $\operatorname{spin}(8)$ triality cries out for physical interpretation, so far in vain.

The proposed probability space consists of all tensors constructed inductively from a finite number of triadic vertices $\gamma$ of (22), by tensor multiplication, linear combination, contraction (connecting two compatible lines), and identification modulo commutation relations of the Yang-Palev kind. Odd lines obey Fermi statistics. Even lines obey the unique Palev statistics induced by this Fermi statistics. 
The outstretched arms of $\gamma$ represent chiral spinors, and the leg represents a vector in the first grade of a Clifford algebra associated with the spinor space. The spinors have exchange parity 1 and the vector exchange parity 0 . All the terminals of $\gamma$ are polarized. Spinors plug only into dual spinors, and dyads only into dyads.

Call the quantum structure whose history probability tensors are so constructed a quantum interaction network. Its probability tensors derive from Feynman diagrams and Penrose spin networks more than Cantor sets.

This diagram algebra will be developed further.

But it begins to seem likely that the three simple physical Lie algebras introduced by Yang into kinematics, Palev into statistics, and Yang-Mills into differential geometry are actually different representations of one. Whether this shows the pernicious influence or the unreasonable effectiveness of mathematics remains to be seen.

\section{Acknowledgments and references}

Jack Schwartz and Martin Davis taught me some of the logical works of Post and Gödel. Roger Penrose and Feynman showed me their spin atomizations of space or space-time. The Lindisfarne lectures of Lynn Margulis introduced me to symbiogenesis. Tchavdar Palev discussed his statistics with me. David Bohm convinced me that the mathematics in mathematical physics is best regarded as an extension of natural language. Frank (Tony) Smith shared his knowledge of Lie and Clifford algebras with me. James Baugh, David Edwards, Shlomit Ritz Finkelstein, Andry Galiautdinov, Dennis Marks, Zbigniew Oziewicz, Heinrich Saller, Sarang Shah, and Mohsen Shiri-Garakani provided many helpful discussions and corrections. I thank them all deeply.

This paper is dedicated to Jack Schwartz.

\section{References}

1. D.Bohm. The Special Theory of Relativity. W. A. Benjamin, New York (1962). Especially the philosophical appendix.

2. F. Bopp and R. Haag. Über die Möglichkeit von Spinmodellen. Zeitschrift für Naturforschung 5a:644 (1950).

3. P. A.M. Dirac. Spinors in Hilbert Space. Plenum, New York (1974)

4. R. P. Feynman. Personal communication of 1961. Feynman wrote much this formula ca. 1941. It may have appeared in a footnote, but we were unable to find it.

5. D. R. Finkelstein. Palev statistics. In V. K. Dobrev, editor, Lie Theory and its Applications in Physics: IX International Workshop Springer 2012.

6. M. Gerstenhaber. On the deformation of rings and algebras. Annals of Mathematics 79:59-103 (1964)

7. L. Margulis and D. Sagan. Acquiring Genomes: A Theory Of The Origin Of Species. Basic Books (2002).

8. T. D. Palev. Lie algebraical aspects of the quantum statistics. Unitary quantization (A-quantization). Joint Institute for Nuclear Research Preprint JINR E17-10550. Dubna (1977). hep-th/9705032.

9. R. Penrose. Angular momentum: an approach to combinatorial space-time. In T. Bastin (ed.), Quantum Theory and Beyond, 151-180. Cambridge 1971. Penrose kindly shared much of this seminal work with me ca. 1960.

10. H. Saller. Operational quantum theory I. Nonrelativistic structures. Springer, New York (2006).

11. J. Schwartz. The pernicious influence of mathematics on science. In Logic, Methodology and Philosophy of Science, Proceeding of the 1960 International Congress, 44:356-360 (1966).

12. I. E. Segal. A class of operator algebras which are determined by groups. Duke Mathematical Journal 18:221-265 (1951). Especially $\S 6 \mathrm{~A}$.

13. H. Simon. The Architecture of Complexity. Proceedings of the American Philosophical Society 106:6 (1962) 
14. H. P. Snyder. Quantized space-time. Physical Review 71:38 (1947)

15. E. C. G. Stückelberg. Quantum theory in real Hilbert space. Helvetica Physica Acta 33:727-752 (1960)

16. Wigner, E. P. The unreasonable effectiveness of mathematics in the natural sciences. Communications on Pure and Applied Mathematics 13: 114 (1960).

17. C.N. Yang. On Quantized Space-Time. Physical Review 72:874 (1947). 